International Journal of Pure and Applied Mathematics

Volume 110 No. 1 2016, 125-141

ISSN: 1311-8080 (printed version); ISSN: 1314-3395 (on-line version)

url: http://www.ijpam.eu

doi: 10.12732/ijpam.v110i1.13

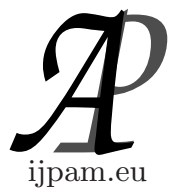

\title{
MODELLING THE IMPACT OF DELAY IN MEDIA COVERAGE ON THE DYNAMICS OF EBOLA IN A LOGISTICALLY GROWING POPULATION
}

\author{
Onyango Brenda Achieng ${ }^{1 \S}$, Owuor George Lawi ${ }^{2}$, Kagendo Joyce Nthiiri ${ }^{3}$ \\ $1,2,3$ Department of Mathematics \\ Masinde Muliro University of Science and Technology \\ P.O. Box 190-50100, Kakamega, KENYA
}

\begin{abstract}
Ebola virus disease(EVD) is a zoonotic filovirus caused by an RNA virus of the family filoviridae and genus Ebolavirus. Its mode of transmission is well known. The use of media in awareness campaigns is critical in controlling the spread of the outbreak. Media usage as an optimal control measure has been studied by a number of scholars, however, they have not considered the impact of delay in media coverage. In this paper, we formulate a mathematical model based on a system of ordinary differential equations (ODEs) with time delay incorporated in a non-linear incidence term to investigate the impact of delay in media reporting on the spread of Ebola in a logistically growing population. In particular, we establish the existence of its steady states and the basic reproduction number. Stability analysis of the model is done and numerical simulation carried out to graphically illustrate the long term behavior of the model with respect to media coverage. The obtained findings are significant in the sense that awareness campaigns during outbreaks, effectively done by means of modern communication channels ensure that individuals take precautionary measures as well as encourage people with symptoms to seek immediate medical attention.
\end{abstract}

AMS Subject Classification: 35E21

Key Words: ebola virus disease, delay in media coverage, logistic population

Received: $\quad$ July 18, 2016

Revised: $\quad$ September 17, 2016

Published: October 21, 2016

$\S_{\text {Correspondence author }}$ (c) 2016 Academic Publications, Ltd. url: www.acadpubl.eu 


\section{Introduction}

The notations used in this paper are standard and can be obtained from the references. The great majority of past Ebola outbreaks in humans have been linked to three Ebola strains namely; EBOV, SUDV, and BDBV. Ebola Virus Disease (EVD) first appeared in 1976 in two simultaneous outbreaks, one in Nzara, Sudan and the other in Yambuku, Democratic Republic of Congo (DRC). The later occurred in a village near the Ebola river, Pourrut [6], from which the disease takes its name. EVD outbreaks among humans have been associated with direct human exposure to fruit bats-the most likely reservoir of the virus or through contact with intermediate infected hosts, which include gorillas, chimpanzees and monkeys. EBOV is transmitted by direct human to human contact via body fluids or indirect contact with contaminated surfaces.

In Dowell [3], individuals become symptomatic after an average incubation period mostly ranging from 2-21 days and infectiousness is increased during the later stages of the disease. Characteristic symptoms of EVD are nonspecific and include sudden onset of fever, weakness, vomiting, diarrhea, headache,and a sore throat, while only a fraction of the symptomatic individuals present with haemorrhagic manifestations. The fatality rate of EVD ranges from $30 \%$ to $90 \%$ depending on the virus species (WHO) [10].

An epidemic that started in early 2014 and which eventually spread to four West African countries (Guinea, Liberia, Sierra Leone, and Nigeria), infected over 2000 individuals of some of the most impoverished and logistically challenged countries of the world as of August 2014 (WHO) [11]. The size of this EBOV outbreak far surpasses the total number of cases reported for all previous Ebola outbreaks combined. Therefore WHO declared the epidemic a public health emergency of international concern (WHO) [10]. The large intermixing populations has facilitated the spread of the infection, but a large epidemic was not inevitable. Currently there is no cure for Ebola but a WHO news release from Geneva, in July 2015 indicate that the world is on the verge of an effective Ebolavirus vaccine with interim results showing $100 \%$ efficacy in individuals (WHO) [10]. However in Muyembe [5], treatment against EVD mainly consists of providing medical care based on symptomatic therapy to maintain the vital respiratory, cardio-vascular and renal functions.

Several mathematical models have been proposed to investigate the impact of media reporting on the transmission dynamics of infectious diseases for instance Cui et al. [2] and Huitao et al. [4]. However the models are too general. It is worthy to note that media coverage plays an important role in prompting action from relevant authorities to contain an outbreak as well as eliciting pre- 
cautionary measures among the susceptible. Economic and sociocultural factors together with the delay in identifying and or reporting an outbreak in an urban setting may hinder timely and effective implementation of control efforts.

\section{Model Description and Formulation}

\subsection{The model}

In our model, the human population is subdivided into classes of susceptible $\mathrm{S}(\mathrm{t})$, infected $\mathrm{I}(\mathrm{t})$, and recovered $\mathrm{R}(\mathrm{t})$. The susceptible population is recruited logistically at the rate $r$, while the population can decrease due to natural deaths at a rate $\mu$. Infected individuals die naturally or as a result of infection at the rate $\sigma$, infected individuals recover at the rate $\delta$ and are also removed as a result of natural death. $m$ - is the effect of media coverage and $g(I)-$ is the force of infection.

The total population is given by;

$$
N(t)=S(t)+I(t)+R(t)
$$

The resulting model is represented by the flow diagram below.

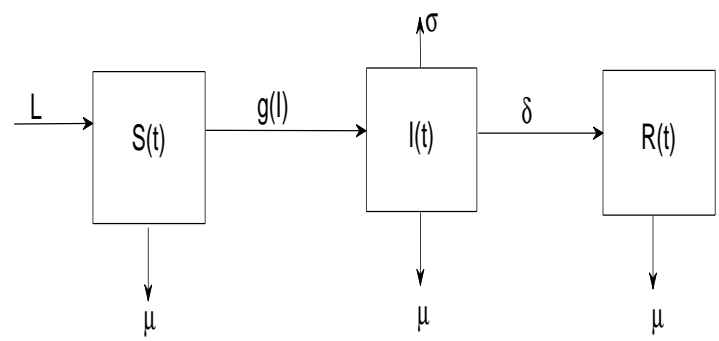

Figure 1: Model Flow Chart

Where $L=r S\left(1-\frac{S}{K}\right)$. The system of differential equations describing the dynamics of the model is as follows;

$$
\frac{d S}{d t}=r S\left(1-\frac{S}{K}\right)-g(I) S-\mu S
$$




$$
\begin{aligned}
\frac{d I}{d t} & =g(I) S-(\mu+\sigma+\delta) I \\
\frac{d R}{d t} & =\delta I-\mu R
\end{aligned}
$$

where

$$
g(I)=\left(\beta_{1}-\frac{\beta_{2} I(t-\tau)}{m+I(t-\tau)}\right) I
$$

where $\tau>0$ is a time delay representing the latent period of media coverage. The term $\frac{\beta_{2} I}{m+I}$ measures the effect of reduction of the contact rate when infectious individuals are reported in the media. The function $\frac{I}{m+I}$ is a continuous bounded function which takes into account disease saturation or psychological effects. Substituting eqn 3 into eqn 2 yields;

$$
\begin{aligned}
\frac{d S(t)}{d t} & =r S\left(1-\frac{S}{K}\right)-\left(\beta_{1}-\frac{\beta_{2} I(t-\tau)}{m+I(t-\tau)}\right) S(t) I(t)-\mu S(t) \\
\frac{d I(t)}{d t} & =\left(\beta_{1}-\frac{\left.\beta_{2} I(t-\tau)\right)}{m+I(t-\tau)}\right) S(t) I(t)-(\mu+\sigma+\delta) I(t) \\
\frac{d R(t)}{d t} & =\delta I(t)-\mu R(t)
\end{aligned}
$$

Suppose that the initial condition for the system 4 takes the form:

$$
\begin{aligned}
S(\theta)=\phi_{1}(\theta), I(\theta)=\phi_{2}(\theta) & R(\theta)=\phi_{3}(\theta), \\
\mid & \phi_{i}(\theta) \geq 0, \theta \in[-\tau, 0], \phi_{i}(0)>0: i=1,2,3,
\end{aligned}
$$

such that $\phi=\left(\phi_{1}, \phi_{2}, \phi_{3}\right)$ are defined in the Banach Space of continuous functions mapping the interval $[-\tau, 0] \mapsto \mathbb{R}^{3}$

\section{Model Analysis}

It can be shown that the solutions of the model are positive and bounded for $t \geq 0$ and is therefore biologically meaningful.

\subsection{Existence of Disease Free Equilibrium (DFE) point}

The disease free equilibrium is defined as the point at which no Ebola disease is present in the population. 
Proposition 1. For the model in system 4 there always exists disease free equilibrium point denoted $E_{0}$, given by $E_{0}=\left(K\left(1-\frac{\mu}{r}\right), 0,0\right)$

Proof. At DFE $I=0$ and $R=0$, Therefore considering the first equation in system 4 and replacing $I=0$ yields;

$$
S=K\left(1-\frac{\mu}{r}\right)
$$

Therefore the DFE $E_{0}\left(S^{0}, I^{0}, R^{0}\right)=\left(K\left(1-\frac{\mu}{r}\right), 0,0\right)$

\subsection{Existence of Endemic Equilibrium (EE) point}

This is the state where the disease cannot be totally eradicated but remains in the population. The existence of this state is shown using the definition and the theorem below.

The basic reproduction number $\left(R_{0}\right)$ :-Is defined as the average number of secondary infections due to a single infectious individual introduced in a fully susceptible population. If $R_{0}<1$ it means the disease is contained in the population and $R_{0}>1$ means the disease is persistent in the population.

The constant $R_{O}$ is determined by the method of next generation matrix approach [9]. The reproduction number $R_{0}=\rho\left(F V^{-1}\right)$, is the spectral radius of the matrix $F V^{-1}$. Therefore;

$$
R_{0}=\frac{\beta_{1} K\left(1-\frac{\mu}{r}\right)}{\mu+\sigma+\delta}
$$

Theorem 1. Assume that $2 \beta_{1} \beta_{2} \geq \beta_{1}^{2}+\beta_{2}(r-\mu)$ and $2 r \mu \leq 1$ then the model has atleast one unique endemic equilibrium $E^{*}\left(S^{*}, I^{*}, R^{*}\right)$ whenever $R_{0}>1$.

Proof. Using the third equation in system $4, \mu R=\delta I$

$$
R^{*}=\frac{\delta I^{*}}{\mu}>0, \forall I^{*}>0
$$

Using equation two of system 4

$$
\begin{aligned}
& \left(\beta_{1}-\frac{\beta_{2} I}{m+I}\right) S I=(\mu+\sigma+\delta) I \\
& \frac{(m+I) \beta_{1}-\beta_{2} I}{m+I} S=(\mu+\sigma+\delta)
\end{aligned}
$$




$$
S^{*}=\frac{\left(m+I^{*}\right)(\mu+\sigma+\delta)}{\left(m+I^{*}\right) \beta_{1}-\beta_{2} I^{*}}>0, \forall I^{*}>0, \beta_{1}>\beta_{2}
$$

Substituting 7 into equation 1 of system 4 at the equilibrium point, we obtain

$$
\begin{gathered}
r\left(\frac{(m+I)(\mu+\sigma+\delta)}{(m+I) \beta_{1}-\beta_{2} I}\right)\left(1-\frac{1}{K}\left(\frac{(m+I)(\mu+\sigma+\delta)}{(m+I) \beta_{1}-\beta_{2} I}\right)\right)- \\
\left(\beta_{1}-\frac{\beta_{2} I}{m+I}\right)\left(\frac{(m+I)(\mu+\sigma+\delta)}{(m+I) \beta_{1}-\beta_{2} I}\right) I-\mu\left(\frac{(m+I)(\mu+\sigma+\delta)}{(m+I) \beta_{1}-\beta_{2} I}\right)=0
\end{gathered}
$$

Since $S^{*} \neq 0$ it follows from equation 8 that;

$$
r\left(1-\frac{1}{K}\left(\frac{(m+I)(\mu+\sigma+\delta)}{(m+I) \beta_{1}-\beta_{2} I}\right)\right)-\left(\beta_{1}-\frac{\beta_{2} I}{m+I}\right) I-\mu=0
$$

Equation 9 can be simplified as

$$
A I^{2}+B I-C=0
$$

where

$$
\begin{gathered}
A=K \beta_{1}(r-\mu)+K\left(2 \beta_{1} \beta_{2}-\beta_{1}^{2}-\beta_{2}(r-\mu)-\beta_{2}^{2}\right) \\
B=2 m r(\mu+\sigma+\delta)\left(\frac{\beta_{1} K(r-\mu)}{r(\mu+\sigma+\delta)}-\frac{1}{2 m r}\right)+m K\left(2 \beta_{1} \beta_{2}-\beta_{1}^{2}-\beta_{2}(r-\mu)\right) \\
=2 m r(\mu+\sigma+\delta)\left(R_{o}-\frac{1}{2 m r}\right)+m K\left(2 \beta_{1} \beta_{2}-\beta_{1}^{2}-\beta_{2}(r-\mu)\right) \\
C=r m^{2} K \beta_{1}+m(\mu+\sigma+\delta)-m K \beta_{1}-m^{2} K \beta_{1}
\end{gathered}
$$

with

$$
E^{*}=\left(S^{*}, I^{*}, R^{*}\right)=\left(\frac{\left(m+I^{*}\right)(\mu+\sigma+\delta)}{\left(m+I^{*}\right) \beta_{1}-\beta_{2} I^{*}}, I^{*}, \frac{\delta I^{*}}{\mu}\right)
$$

It can be deduced that $B>0$, whenever $R_{O}>1$ and the assumptions hold. Equation 10 can therefore be expressed as $-A I^{2}+B I-C=0$ or $A I^{2}+B I-C=$ 0 . In both cases, there exists $I^{*}>0$, hence the model has a positive endemic equilibrium.

\subsection{Local Stability of the DFE}

The stability of equilibrium point is closely linked to the basic reproduction number of the model under study.

Proposition 2. For any time delay $\tau \geq 0$, the disease free equilibrium $E_{0}=$ $\left(K\left(1-\frac{\mu}{r}\right), 0,0\right)$ of system 4 is asymptotically stable when $R_{0}<1$ and unstable when $R_{0}>1$ 
The Jacobian matrix of the linearized system of the system 4 is;

$$
J=\left(\begin{array}{ccc}
\mu-r & \beta_{1} S & 0 \\
0 & -(\mu+\sigma+\delta)+\beta_{1} S & 0 \\
0 & \delta & -\mu
\end{array}\right)
$$

Proof. Evaluating the Jacobian matrix 12 at DFE $E_{0}$, where $E_{0}\left(S^{0}, I^{0}, R^{0}\right)=$ $\left(K\left(1-\frac{\mu}{r}\right), 0,0\right)$ yields;

$$
J_{D F E}=\left(\begin{array}{ccc}
\mu-r & -R_{0}(\mu+\sigma+\delta) & 0 \\
0 & R_{0}-1 & 0 \\
0 & \delta & -\mu
\end{array}\right)
$$

To compute the eigenvalues, we solve;

$$
|J|=\left|\begin{array}{ccc}
\mu-r-\lambda & -R_{0}(\mu+\sigma+\delta) & 0 \\
0 & R_{0}-1-\lambda & 0 \\
0 & \delta & -\mu-\lambda
\end{array}\right|=0
$$

Evaluating 14, we obtain the roots of $\lambda$ to be; $\lambda=\left(\mu-r, R_{0}-1,-\mu\right)$. For local asymptotic stability, all real parts of $\lambda$ should be negative. The last root is negative, the root $\lambda=R_{0}-1$ is negative if and only if $R_{0}<1$, and the root $\lambda=\mu-r$ is negative since the population growth rate is assumed to be greater than the natural death rate.

\subsection{Global Stability of the Disease Free Equilibrium}

To study the global stability of DFE we use the technique by Castillo Chavez [1]. This requires that system 4 be written in the form;

$$
\begin{gathered}
\frac{d X}{d t}=F(X, Z) \\
\frac{d Z}{d t}=G(X, Z), G(X, 0)=0
\end{gathered}
$$

where $X \in \mathbb{R}^{1}$ and $\mathrm{X}=(\mathrm{S})$ denotes the number of uninfected individuals and $Z \in \mathbb{R}^{1}$, where $\mathrm{Z}=(\mathrm{I})$ denotes the number of infected individuals. The following conditions below must be met to guarantee global asymptotic stability.

$$
\begin{gathered}
\frac{d X}{d t}=F(X, 0), X^{*}(G . A . S) \\
G(X, Z)=A Z-\widehat{G}(X, Z), \widehat{G}(X, Z) \geq 0 \forall(X, Z) \in \Gamma
\end{gathered}
$$


Where $A=D_{Z} G\left(X^{*}, Z\right)$ is an M-matrix and $\Gamma$ is the region where the model makes biological sense.

Theorem 2. The DFE $E_{0}$ of system 4 is globally asymptotically stable if $R_{0}<1$ and unstable if $R_{0}>1$

Proof. ; The proof is done using the theorem below,

Theorem 3. We observe that the 1st two equations of system 4 are independent of $R$, we therefore rewrite system 4 as:-

$$
\begin{gathered}
\frac{d S(t)}{d t}=r S\left(1-\frac{S}{K}\right)-\left(\beta_{1}-\frac{\beta_{2} I(t-\tau)}{m+I(t-\tau)}\right) S(t) I(t)-\mu S(t) \\
\frac{d I(t)}{d t}=\left(\beta_{1}-\frac{\left.\beta_{2} I(t-\tau)\right)}{m+I(t-\tau)}\right) S(t) I(t)-(\mu+\sigma+\delta) I(t)
\end{gathered}
$$

Therefore;

$$
\begin{gathered}
\frac{d X}{d t}=F(X, Z)=r S\left(1-\frac{S}{K}\right)-\mu S \\
G(X, Z)=A Z-\widehat{G}(X, Z)=
\end{gathered}
$$

right hand side of equation two in system 4 .

$$
A=D_{Z} G\left(X^{*}, 0\right)
$$

-differentiating right hand side of second equation in system 4 with respect to I.

$$
\frac{d I}{d t}=\beta_{1} S-(\mu+\sigma+\delta)
$$

Therefore

$$
\begin{gathered}
A Z=\left(\beta_{1} S-(\mu+\sigma+\delta)\right) I \\
\widehat{G}(X, Z)=A Z-G(X, Z) \\
=\left(\beta_{1} S-(\mu+\sigma+\delta)\right) I-\left(\left(\beta_{1}-\frac{\beta_{2} I(t-\tau)}{m+I(t-\tau)}\right) S I-(\mu+\sigma+\delta) I\right) \\
=\left(\frac{\beta_{2} I(t-\tau)}{m+I(t-\tau)}\right) S I>0
\end{gathered}
$$

Since $\widehat{G}(X, Z) \geq 0$, the DFE of system 4 is globally asymptotically stable. 


\subsection{Local Stability of Endemic Equilibrium (EE) point}

The Jacobian matrix at the endemic equilibrium $E^{*}$ can be expressed as:

$$
\operatorname{det}\left|\begin{array}{ccc}
\lambda-a_{1} & -a_{2}-a_{5} e^{-\lambda \tau} & 0 \\
-a_{3} & \lambda-a_{4}+a_{5} e^{-\lambda \tau} & 0 \\
0 & -\delta & \lambda-a_{6}
\end{array}\right|=0
$$

where

$$
\begin{gathered}
a_{1}=r-\frac{2 r S^{*}}{K}-\beta_{1} I^{*}+\left(\frac{\beta_{2} I^{*}}{m+I^{*}}\right)-\mu \\
a_{2}=-\beta_{1} S^{*}+\left(\frac{\beta_{2} S^{*} I^{*}}{m+I^{*}}\right) \\
a_{3}=\beta_{1} I^{*}-\left(\frac{\beta_{2} I^{* 2}}{m+I^{*}}\right) \\
a_{4}=-(\mu+\sigma+\delta)+\beta_{1} S^{*}-\left(\frac{\beta_{2} S^{*} I^{*}}{m+I^{*}}\right) \\
a_{5}=m\left(\frac{\beta_{2} S^{*} I^{*}}{m+I^{* 2}}\right) \\
a_{6}=-\mu
\end{gathered}
$$

The linearized system of 4 at a positive steady state gives the following characteristic equation $\left(\lambda-a_{1}\right)\left(\lambda-a_{4}+a_{5} e^{-\lambda \tau}\right)\left(\lambda-a_{6}\right)$ which gives

$$
\lambda^{3}+\lambda^{2} b_{1}+\lambda b_{2}+b_{3}+\left(\lambda^{2} b_{4}+\lambda b_{5}+b_{6}\right) e^{-\lambda \tau}
$$

where $b_{1}=-\left(a_{1}+a_{4}+a_{6}\right), b_{2}=a_{1} a_{6}+a_{4} a_{6}+a_{1} a_{4}, b_{3}=-a_{1} a_{4} a_{6}, b_{4}=a_{5}$, $b_{5}=-a_{5}\left(a_{1}+a_{6}\right)$ and $b_{6}=a_{1} a_{5} a_{6}$.

Which is a third degree exponential polynomial equation. It is well known that the steady state is stable if all eigenvalues of the exponential polynomial equation have negative real parts, and unstable if atleast one root has a positive real part.

Suppose $\lambda=i \omega, \omega>0$ is a root of equation 17 . We substitute $\lambda=i \omega$ into 17 to derive

$$
-\omega^{3} i-b_{1} \omega^{2}+b_{2} \omega i+b_{3}+\left(-b_{4} \omega^{2}+b_{5} \omega i+b_{6}\right)(\cos (\omega \tau)-i \sin (\omega \tau))=0
$$

Separating the real and imaginary parts gives

$$
b_{1} \omega^{2}-b_{3}=\left(b_{6}-b_{4} \omega^{2}\right) \cos (\omega \tau)+b_{5} \omega \sin (\omega \tau)
$$




$$
-\omega^{3}+b_{2} \omega=\left(b_{6}-b_{4} \omega^{2}\right) \sin (\omega \tau)-b_{5} \omega \cos (\omega \tau)
$$

Squaring and adding both equations in 18, we obtain

$$
\omega^{6}+\left(b_{1}^{2}-b_{4}^{2}-2 b_{2}\right) \omega^{4}+\left(b_{2}^{2}-2 b_{1} b_{3}-b_{5}^{2}+2 b_{4} b_{6}\right) \omega^{2}+\left(b_{3}^{2}-b_{6}^{2}\right)
$$

Let $z=\omega^{2}$ and $p=b_{1}^{2}-b_{4}^{2}-2 b_{2}, q=b_{2}^{2}-2 b_{1} b_{3}-b_{5}^{2}+2 b_{4} b_{6}$ and $r=b_{3}^{2}-b_{6}^{2}$. Then, equation 18 becomes

$$
z^{3}+p z^{2}+q z+r=0
$$

Claim 1. If $r<0$, then equation 20 has at least one positive root

Proof. Denote

$$
h(z)=z^{3}+p z^{2}+q z+r
$$

So $h(0)=r<0$ and $\lim _{z \rightarrow \infty} h(z)=\infty$. Hence, there exists a $z_{0} \in(0, \infty)$ so that $h\left(z_{0}\right)=0$. This completes the proof.

Claim 2. If $r \geq 0$, then, the necessary condition for equation 20 to have a positive real root is

$$
\triangle=p^{2}-3 q \geq 0
$$

Proof. From equation 21, we have

$$
\begin{gathered}
\frac{d h(z)}{d z}=3 z^{2}+2 p z+q \\
3 z^{2}+2 p z+q=0
\end{gathered}
$$

The roots of equation 23 can be expressed as

$$
z_{1,2}=\frac{-2 p \pm \sqrt{4 p^{2}-12 q}}{6}=\frac{-p \pm \sqrt{\triangle}}{3}
$$

If $\triangle<0$, then eqn 23 does not have real roots, so the function $\mathrm{h}(\mathrm{z})$ is monotone increasing in $\mathrm{z}$. It follows from $h(0)=r \geq 0$ that equation 20 has no positive real roots.

Claim 3. If $r \geq 0$, then equation 20 has positive roots if and only if $z_{1}>0$ and $h\left(z_{1}\right) \leq 0$ 
Proof. Assume that either $z_{1} \leq 0$ or $z_{1}>0$ and $h\left(z_{1}\right)>0$. If $z_{1} \leq 0$, since $h(z)$ is increasing for $z \geq z_{1}$ and $h(0)=r \geq 0$, it follows that $h(z)$ has no positive real zeros. If $z_{1}>0$ and $h\left(z_{1}\right)>0$, since $z_{2}=\frac{-p-\sqrt{\triangle}}{3}$ is the local maximum value, it follows that $h\left(z_{1}\right)<h\left(z_{2}\right)$ hence, by $h(0)=r \geq 0$, we know that $h(z)$ does not have positive real zeros.

Lemma 1. The claims 1,2 and 3 above can be summarized as follows;

(i) If $r<0$, then equation 20 has atleast one positive root.

(ii) If $r \geq 0$ and $\triangle=p^{2}-3 q<0$, then equation 20 has no positive roots.

(iii) If $r \geq 0$, then equation 20 has positive roots if and only if $z_{1}=\frac{-p+\sqrt{\triangle}}{3}>0$ and $h\left(z_{1}\right) \leq 0$

Suppose that equation 20 has positive roots, without loss of generality, we assume that it has three positive roots denoted by $z_{1}, z_{2}, z_{3}$. Then equation 19 has three positive roots, say; $\omega_{1}=\sqrt{z_{1}}, \omega_{2}=\sqrt{z_{2}}, \omega_{3}=\sqrt{z_{3}}$ From 18 we have;

$$
\begin{gathered}
\sin \omega \tau=\frac{b_{4} \omega^{5}-b_{2} b_{3} \omega^{3}-b_{6} \omega^{2}+b_{2} b_{6} \omega+b_{5} \omega\left(b_{1} \omega^{2}-b_{3}\right)}{b_{5}^{2} \omega^{2}-b_{4} \omega^{2}\left(b_{6}-b_{4} \omega^{2}\right)+b_{6}\left(b_{6}-b_{4} \omega^{2}\right)} \\
\tau_{k}^{j}=\frac{1}{\omega_{k}}\left\{\sin ^{-1}\left(\frac{b_{4} \omega_{k}^{5}-b_{2} b_{3} \omega_{k}^{3}-b_{6} \omega_{k}^{2}+b_{2} b_{6} \omega_{k}+b_{5} \omega_{k}\left(b_{1} \omega_{k}^{2}-b_{3}\right)}{b_{5}^{2} \omega_{k}^{2}-b_{4} \omega_{k}^{2}\left(b_{6}-b_{4} \omega_{k}^{2}\right)+b_{6}\left(b_{6}-b_{4} \omega_{k}^{2}\right)}\right)+2 \pi j\right\}
\end{gathered}
$$

$k=1,2,3 ;$ and $j=0,1,2 \ldots \ldots$. , then $\pm \omega_{k}$ is a pair of purely imaginary roots of equation 17 with $\tau=\tau_{k}^{j}, k=1,2,3 ; j=0,1, \ldots \ldots \ldots \ldots$ then $\lim _{j \rightarrow \infty} \tau_{k}^{j}=\infty$ $k=1,2,3$ Therefore we define

$$
\tau_{0}=\tau_{k 0}^{j 0}=\min _{1 \leq k \leq 3, j \geq 1} \tau_{k}^{j}, \omega_{0}=\omega_{k 0}
$$

From lemma 1, when $\tau=0$, the endemic equilibrium $E^{*}$ is stable if $R_{0}>1$, we use a result from Ruan and Wei [7] using Rouche's theorem to analyze equation 17.

Lemma 2. Consider the exponential polynomial

$$
\begin{gathered}
P\left(\lambda, e^{-\lambda \tau_{i}}, \ldots \ldots . . e^{-\lambda \tau_{m}}\right)=\lambda^{n}+p_{1}^{0} \lambda^{n-1}+\ldots .+p_{n-1}^{0} \lambda+p_{n}^{0}+ \\
{\left[p_{1}^{1} \lambda^{n-1}+\ldots \ldots+p_{n-1}^{1} \lambda+p_{n}^{1}\right] e^{-\lambda \tau_{i}}+\ldots \ldots+\left[p_{1}^{m-1} \lambda^{n-1}+\ldots . .+p_{n-1^{m-1}} \lambda+p_{n}^{m-1}\right] e^{-\lambda \tau_{m}}}
\end{gathered}
$$


where $\tau_{i} \geq 0(i=1,2, \ldots ., m)$ and $p_{j}^{i}(i=0,1, \ldots ., m-1 ; j=1,2, \ldots . ., n)$ are constants. As $\left(\tau_{1}, \tau_{2}, \ldots \ldots . \tau_{m}\right)$ vary the sum of the orders of the zeros of $p\left(\lambda, e^{-\lambda \tau_{i}}, \ldots . ., e^{-\lambda \tau_{m}}\right)$ on the open right half plane can change only if a zero appears on or crosses the imaginary axis.

Applying Lemma 1 and 2, we obtain;

Lemma 3. For a third degree exponential polynomial equation 17, we have the following;

(i) If $r \geq 0$ and $\triangle=p^{2}-3 q<0$, then all the roots of equation 17 have negative real parts for all $\tau \geq 0$

(ii) If $r<0$ or $r \geq 0, z_{1}>0$ and $h\left(z_{1}\right) \leq 0$, then all the roots of equation 17 have negative real parts when $\tau \epsilon\left[0, \tau_{0}\right)$

Let

$$
\lambda(\tau)=\alpha(\tau)+i \omega(\tau)
$$

be the root of equation 17 satisfying $\alpha\left(\tau_{0}\right)=0, \omega\left(\tau_{0}\right)=\omega_{0}$ To guarantee that $\pm \omega_{0} i$ are simple purely imaginary roots of equation 17 with $\tau=\tau_{0}$ and $\lambda(\tau)$ satisfies the transversality condition which leads to;

Lemma 4. Suppose that $z_{k}=\omega_{k}^{2}$ and $h^{\prime}\left(z_{k}\right) \neq 0$, then $\frac{R \lambda\left(\tau_{k}^{j}\right)}{d \tau} \neq 0$ and $\frac{R \lambda\left(\tau_{k}^{j}\right)}{d \tau}$ and $h^{\prime}\left(z_{k}\right)$ have the same sign

Proof. Consider the characteristic equation 17 of system 4 , from $17, b_{1}=$ $-\left(a_{1}+a_{4}+a_{6}\right), b_{2}=a_{1} a_{6}+a_{4} a_{6}+a_{1} a_{4}, b_{3}=-a_{1} a_{4} a_{6}, b_{4}=a_{5}, b_{5}=-a_{5}\left(a_{1}+a_{6}\right)$ and $b_{6}=a_{1} a_{5} a_{6}$

$$
\begin{gathered}
p=b_{1}^{2}-b_{4}^{2}-2 b_{2} \\
=\left[-\left(a_{1}+a_{4}+a_{6}\right)\right]^{2}-a_{5}^{2}-2\left(a_{1} a_{6}+a_{4} a_{6}+a_{1} a_{4}\right) \\
q=b_{2}^{2}-2 b_{1} b_{3}-b_{5} \\
=\left(a_{1} a_{6}+a_{4} a_{6}+a_{1} a_{4}\right)^{2}-2\left(\left(a_{1}+a_{4}+a_{6}\right)\left(a_{1} a_{4} a_{6}\right)\right)-\left(a_{5}\left(a_{1}+a_{6}\right)\right)^{2} \\
r=b_{3}^{2}-b_{6}^{2} \\
a_{1}^{2} a_{4}^{2} a_{6}^{2}-a_{1}^{2} a_{5}^{2} a_{6}^{2}>0
\end{gathered}
$$

Therefore we can compute; $\triangle=p^{2}-3 q, h(z)=z^{3}+p z^{2}+q z+r, z_{1}^{*}=$ $\frac{1}{3}(-p+\sqrt{\triangle})$ When $\tau=0,17$ becomes;

$$
\lambda^{3}+\lambda^{2}\left(b_{1}+b_{4}\right)+\lambda\left(b_{2}+b_{5}\right)+\left(b_{3}+b_{6}\right)
$$


Applying lemmas 1,2,3 and 4 to 17, we have the following theorems: then;

Theorem 4. Let $\tau_{k}^{j}$ and $\tau_{0}$ be defined by 25 and 26. Suppose that $g(I)>0$,

(i) If $r \geq 0$ and $\triangle=p^{2}-3 q<0$, then all roots of equation 17 have negative real parts for all $\tau \geq 0$.

(ii) If $r<0$ or $r \geq 0, z_{1}>0$ and $h\left(z_{1}\right)<0$, then all roots of equation 17 have negative real parts for $\tau \in\left[0, \tau_{0}\right)$.

(iii) If the conditions of (ii) are satisfied, $\tau=\tau_{0}$, and $h^{\prime}\left(z_{0}\right) \neq 0$, then $\pm \omega_{0} i$ is a pair of simple purely imaginary roots of equation 17 and all other roots have negative real parts. Moreover $\left(\frac{d}{d \tau}\right) \operatorname{Re} \lambda\left(\tau_{0}\right)>0$

Theorem 5. Let $\tau_{k}^{j}$ and $\tau_{0}$ be defined by 25 and 26. Suppose that $g(I)>0$, then;

(i) If $\triangle \leq 0$, then 17 has all roots with negative real parts for all $\tau \geq 0$, and the equilibrium 11 of the system 4 is stable

(ii) If $\triangle>0, z_{1}^{*}>0$ and $h\left(z_{1}^{*}\right) \leq 0,17$ has all roots with negative real parts for $\tau \in\left[0, \tau_{0}\right)$ and the equilibrium 11 of the system 4 is stable.

(iii) If the conditions of (ii) are satisfied, and $h^{\prime}\left(z_{k}\right) \neq 0$, then system 4 exhibits a Hopf bifurcation at the equilibrium 11 for $\tau=\tau_{k}$

\section{Numerical Simulation}

We carry out numerical simulations to illustrate the long term dynamics of the system. The parameter values used are in the table below and have earlier been defined in the text.

\subsection{Simulation and Interpretation}

For numerical simulation of Ebola transmission of the system 4, we use the following initial populations. The time $\mathrm{t}$ in the simulation is in days. $S_{0}=$ $200, I_{0}=60, R_{0}=20$ The figure 2 below shows the solution dynamics of system 4 when the reproduction number $R_{0}=0.27026701<1$.

The only stable state is the Ebola free equilibrium $E_{0}$ and is asymptotically stable as shown in proposition 2 . From figure 2 we observe that in the 
Table 1: Data for Ebola Model

\begin{tabular}{|c|c|c|}
\hline \hline Parameters and Variables & Range & Source \\
\hline$r$ & 0.5 & {$[9]$} \\
\hline$k$ & 100 & Estimated \\
\hline$\beta_{1}$ & 0.0002 & Varies \\
\hline$\beta_{2}$ & 0.0018 & Varies \\
\hline$\mu$ & 0.01012 & Estimated \\
\hline$\sigma$ & {$[0.2,0.9] d a y^{-1}$} & {$[10]$} \\
\hline$\delta$ & {$[0.1,0.25] d a y^{-1}$} & {$[4]$} \\
\hline$m$ & 0.5 & Varies \\
\hline
\end{tabular}

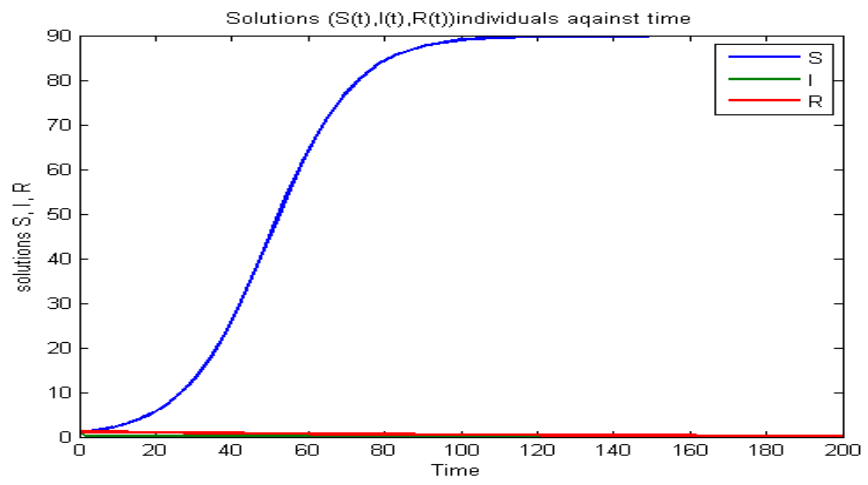

Figure 2: Population growth in the absence of the Disease

presence of time delay, that is, $\tau \geq 0$, the population grows logistically to the carrying capacity. This means that delay in media coverage does not influence the stability of the disease free equilibrium. Solutions converge to Ebola free steady state $E_{0}$, where the disease is swiped out. When $R_{0}=0.27026701949$, that is, there is no infection of Ebola in the population and in the presence of time delay, the infective will decline to the disease free equilibrium.

When $R_{0}>1$, that is in the presence of the disease and the delay parameter $\tau>0$ is large, the disease takes a longer time to be eliminated in the population, hence increasing the force of infection leading to more infective in the population. Delay in appraisal of Ebola disease leads to repeated episodes of the epidemic and therefore containing the disease is difficult as shown in the figure 3 .

However delay in media coverage has no negative impact to the infective in 


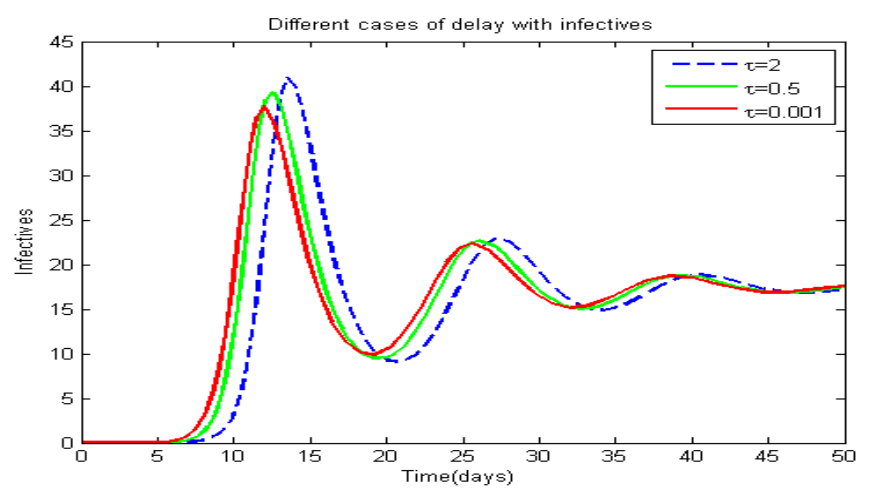

Figure 3: Behavior of Infective in the absence of disease

the absence of infection. The infective drop to the zero mark when there is no disease in the population as shown in figure 4.

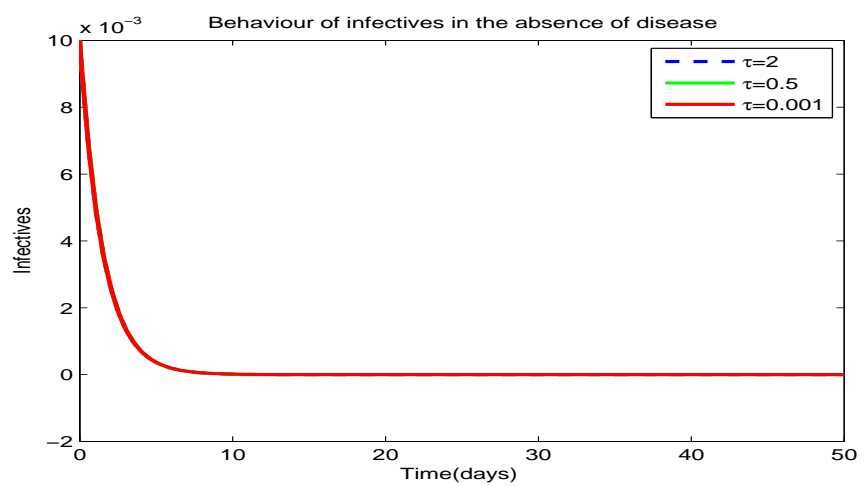

Figure 4: Different cases of delay with infective 


\section{Conclusion}

The main objective of this study is to model the impact of time delay in media coverage on the spread of Ebola in a population with logistic growth. In this study we formulated a mathematical model based on the SIR framework, represented by System (4), in which the population is subject to grow logistically. The model incorporates media coverage with time delay in a nonlinear incidence term. Stability analysis of the disease free equilibrium of System (4) shows that it is globally asymptotically stable, as illustrated by Theorem 2. This means that given any perturbation the disease free equilibrium remains stable. The endemic equilibrium of the System (4), using Theorem 4 and Theorem 5 has been shown to be locally asymptotically stable and exhibits a hopf bifurcation at the equilibrium point. Numerical simulation of the system shows that delay in media reporting does not affect the disease free equilibrium and therefore the population grows logistically. However if the time delay $\tau>0$ with $R_{0}>1$, this is in the presence of the infection then there occurs repeated episodes of the epidemic.

\subsection{Recommendation}

Controlling Ebola virus disease still remains a challenge not only to the West African Countries that were affected in 2014 but the World at large. From the findings of this study it is clear that delay in media coverage on the appraisal of Ebola disease leads to increased cases of the infection, therefore health practitioners and media should collaborate for an early appraisal of any epidemic. Furthermore, many studies have been done on different aspects of the disease, however much still needs to be done to uncover the reasons for the repeated occurrence of the disease.

\section{References}

[1] Chavez C.C, Zhilang F and Wenzhang H. On the computation of $\mathrm{R}_{0}$ and its role on global stability, https://ecommons.cornell.edu/bitstream/1813/32146/BU-1553-M-pdf.

[2] Cui J, Sun Y and Zhu H., The impact of media on the control of infectiousdiseases. Journal of Dynamics and Differential Equations, (2008), vol.20, no.1, 31-53, doi: 10.1007/s10884007-9075-0.

[3] Dowell S F , Mukunu R, Ksiazek T.G, Khan A.S, Rollin P.E et al. Transmission of Ebola hemorrhagic fever: a study of risk factors in family members. Eur. J. Epidemiol. (1995), 41, 1-11, doi: 10.1086/514284. 
[4] Huitao Z, Yiping L, Yunxian D. An SIRS Epidemic Model Incorporating Media Coverage with Time Delay. Computational and Mathematical Methods in Medicine 2014, 1-10. Onlinepublication date: 1-Jan-2014, doi.org/10.1155/2014/680743.

[5] Muyembe J.J, Mulanga S, Masumu J, Kemp A, Paweska J.T. Ebola virus outbreaks in Africa: past and present. Onderstepoort Journal of Veterinary Research, (2012); 79 (2), doi: 10.4102/ojvr.v79i2.451.

[6] Pourrut X, Kumulungui B, Wittmann $\mathrm{T}$ et al. The natural history of Ebola virus in Africa, Microbes and Infection. 7,(2005), 1005-1014, doi: 10.1016/j.micinf.2005.04.006.

[7] Ruan S and Wei J. On the zeros of transcendental functions with applications to stability of delay differential equations with two delays, Dynamics of continous, discrete and impulsive systems A. (2003), vol 10, no.6, 863-874.

[8] Stefano M, Marco A, Laura F, Marcelo F.C, Ana P.P et al. Spatiotemporal spread of the 2014 outbreak of Ebola virus disease in Liberia and the effectiveness of nonpharmaceuticalinterventions. A computational modelling analysis, (2015) Published Online, doi: 10.1016/s1473-3099(14)71074-6.

[9] Van den D.P and Watmough J. Reproduction number and sub-threshold endemic equilibria for compartmental models of disease transmission. Math.Biosci. (2002), 180(1-2), 29-48.

[10] WHO Ebola response team, Ebola virus disease in West Africa- The first 9 months of the epidemic and forward projections, N Engl J Med. (2014), 37116, 1481-1495.

[11] Wikipedia, Timeline of reported cases and deaths, Ebola virus epidemic in West Africa, 2014. 
